\title{
Radical prostatectomy for high-risk clinically localized prostate cancer: a prospective single institution series
}

\author{
Anthony J. Koupparis, MD; Jeremy P. Grummet, MD; Antonio Hurtado-Coll, MD; Robert H. Bell, MD; \\ Nicholas Buchan, MD; S. Larry Goldenberg, MD, FRCSC; Martin E. Gleave, MD, FRCSC
}

Cite as: Can Urol Assoc J 2011;5(6):E156-E161; http://dx.doi.org/10.5489/cuaj.10057. Epub 2011 Mar 1.

\section{Abstract}

Objective: The objective of this paper is to report on the pathologic and biochemical progression-free outcomes of patients who underwent radical prostatectomy for high-risk localized prostate cancer.

Methods: Data was collected prospectively from 299 patients who underwent radical prostatectomy for high-risk clinically localized prostate cancer by 2 surgeons at a single institution. High risk was defined as 1 or more of 3 adverse factors: prostate-specific antigen (PSA) $>20$, biopsy Gleason score 8 to 10 and clinical stage T3. PSA recurrence was defined as PSA $>0.4 \mathrm{ng} / \mathrm{mL}$ or any salvage therapy. Results: Median age was 63.3 years (46.1-75.9). Median followup was 4.7 years (range 0.5-17.3 years). PSA at diagnosis was $>20 \mathrm{ng} / \mathrm{mL}$ in $31.4 \%$. Biopsy Gleason score was 8 to 10 in $66.9 \%$. Clinical stage was T3 in $24.4 \% .81 .6 \%$ of patients had a single baseline risk factor, $15.7 \%$ had 2 risk factors and $2.7 \%$ had all 3 risk factors. Neoadjuvant therapy was administered to 184 patients (61.5\%). Pathologic stage was organ-confined in 39.6\%, specimenconfined in $26 \%$, non-specimen-confined in $26.4 \%$, and $8 \%$ had lymph node positive disease. Overall survival, cancer-specific survival and biochemical progression-free survival was $99 \%$, $99.67 \%$ and $70.2 \%$, respectively. Univariate analysis showed that PSA at diagnosis, percentage of cores positive and number of risk factors were predictors of PSA recurrence $(p<0.05)$. Multivariate analysis showed that PSA at diagnosis was an independent predictor of PSA recurrence $(p<0.05)$.

Conclusion: Radical prostatectomy is associated with favourable biochemical progression-free, clinical and overall survival in selected men with high-risk localized prostate cancer, and should therefore be considered an option in these patients. Baseline PSA $>20 \mathrm{ng} / \mathrm{mL}$ is a significant independent predictor of PSA recurrence.

\section{Résumé}

Objectif : L'objectif de cet article est de faire rapport sur les résultats quant à la survie sans progression pathologique et biochimique des patients ayant subi une prostatectomie radicale pour traiter un cancer de la prostate localisé à risque élevé.

Méthodologie : Les données ont été recueillies de manière prospective chez 299 patients ayant subi une prostatectomie radicale réalisée par 2 chirurgiens dans un même établissement pour traiter un cancer de la prostate à risque élevé cliniquement localisé. Un risque élevé était défini comme au moins 1 des 3 facteurs négatifs suivants : taux d'antigène prostatique spécifique (APS) > 20, score de Gleason de 8 à 10 à la biopsie, stade clinique T3. Une récidive avec anomalie de l'APS a été définie comme un taux d'APS $>0,4 \mathrm{ng} / \mathrm{mL}$ ou le recours à tout traitement de sauvetage. Résultats : L'âge médian était de 63,3 ans $(46,1$ à 75,9$)$. Le suivi médian était de 4,7 ans (0,5 à 17,3 ans). Le taux d'APS au moment du diagnostic était $>20 \mathrm{ng} / \mathrm{mL}$ chez $31,4 \%$ des patients. Le score de Gleason à la biopsie était de 8 à 10 dans 66,9\% des cas. Le stade clinique était de T3 dans $24,4 \%$ des cas; $81,6 \%$ des patients présentaient un seul facteur de risque au départ, 15,7\% présentaient 2 facteurs de risque et 2,7\% présentaient les 3 facteurs de risque. Un traitement néoadjuvant a été administré à 184 patients $(61,5 \%)$. Le stade pathologique était confiné à l'organe dans $39,6 \%$ des cas, confiné à l'échantillon dans $26 \%$ des cas, et non confiné à l'échantillon dans $26,4 \%$ des cas; $8 \%$ des patients présentaient une atteinte des ganglions lymphatiques. La survie globale, la survie spécifique au cancer et la survie sans progression biochimique étaient de $99 \%, 99,67 \%$ et 70,2\%, respectivement. L'analyse univariée a montré que le taux d'APS au moment du diagnostic, le pourcentage de carottes biopsiques positives et le nombre de facteurs de risque étaient des facteurs prédictifs de récidive avec anomalie de l'APS ( $p<0,05)$. L'analyse multivariée a montré que le taux d'APS au moment du diagnostic était un facteur prédictif indépendant de récidive avec anomalie de I'APS $(p<0,05)$.

Conclusion : La prostatectomie radicale est associée à une survie sans progression biochimique, une survie clinique et une survie globale favorables chez des patients sélectionnés atteints d'un cancer de la prostate localisé à risque élevé, et devrait être considérée comme une option de traitement chez ces patients. Un taux d'APS au départ $>20 \mathrm{ng} / \mathrm{mL}$ est un facteur de prédiction indépendant significatif de récidive avec anomalie de l'APS.

\section{Introduction}

Widespread use of prostate-specific antigen (PSA) testing has lead to a migration in stage and grade of prostate cancer, with most men presenting with localized disease. ${ }^{1}$ However, $20 \%$ to $35 \%$ of patients still present with high-risk disease based on a PSA $>20 \mathrm{ng} / \mathrm{mL}$, biopsy Gleason score 8 to 10 or clinical stage T3. ${ }^{2}$ 
The optimal treatment for patients with high-risk prostate cancer remains controversial. Traditionally, surgery has been discouraged due to concerns regarding the side effects of radical prostatectomy (RP), high positive surgical margin rates, risk of lymph node metastasis and high PSA recurrence rates. ${ }^{3}$ Furthermore, several randomized trials demonstrate improved clinical disease-free and overall survival (OS) in patients with advanced prostate cancer using radiotherapy (RT) in combination with androgen deprivation therapy (ADT) versus RT alone. ${ }^{4-5}$ As a result, based on epidemiological data from the United States, most men with clinically advanced prostate cancer receive external beam RT in combination with long-term ADT. ${ }^{6}$

Recent surgical series, however, have shown equivalent or better biochemical progression-free (BPFS) and cancerspecific survival (CSS) rates to RT with ADT, as part of a multimodal approach with the use of adjuvant RT where appropriate..$^{7-9}$ In addition, studies report comparable morbidity to RP for lower risk disease. As a result, many practitioners are re-evaluating the role of RP for high-risk clinically localized prostate cancer.

We present our prospective single institution series of $\mathrm{RP}$ as primary definitive local therapy for high-risk prostate cancer as part of a multimodal approach.

\section{Methods}

We examined patient data from our prospectively updated, institution review board-approved database for patients undergoing RP. Patients identified with high-risk disease were operated on by 2 highly experienced surgeons (who perform about 100 RPs per year) between 1994 and 2009. The definition of high-risk disease includes 1 or more of the following factors: PSA $\geq 20 \mathrm{ng} / \mathrm{mL}$, clinical stage T3 or biopsy Gleason score of 8 to 10, according to the National Comprehensive Cancer Network (NCCN) guidelines. ${ }^{10}$ This definition has been shown to be more predictive of PSA recurrence following RP than others. ${ }^{11}$

Patients receiving neoadjuvant ADT, ${ }^{12}$ chemo-hormonal therapy with docetaxel, ${ }^{13}$ or ADT plus OGX-011, ${ }^{14}$ as part of trial protocols, were included. While neoadjuvant ADT reduces positive margin rates, these investigational interventions have not yet been shown to affect PSA recurrence rates. ${ }^{12}$ Patients with follow-ups of less than 6 months were excluded.

Patients were reviewed regularly postoperatively by the operating surgeon. Prostate-specific antigen recurrence was defined as serum PSA $\geq 0.4 \mathrm{ng} / \mathrm{mL}^{15}$ or any PSA if salvage therapy was given.

Baseline parameters, including PSA at diagnosis, percentage of positive biopsy cores, number of high-risk factors $(1,2$ or 3 ) as well as pathological status, were collected and analyzed for PSA recurrence rates using the
Welch 2 sample T-test, Wilcoxon rank sum test and Chisquare analysis. Multivariate analysis was performed using multiple logistic regressions to determine independent predictive factors for PSA recurrence (a $p$ value $<0.05$ was considered statistically significant).

\section{Results}

Of the 2919 patients who underwent RP for clinically localized disease, 299 (9\%) were identified as high-risk. The median age was 63.3 years (range 46.2-75.9) (Table 1).

One-hundred fifteen $(38.5 \%)$ patients underwent RP without neoadjuvant therapy, $155(51.8 \%)$ received ADT only for up to 8 months preoperatively, 16 (5.4\%) and 13 $(4.3 \%)$ received neoadjuvant ADT plus docetaxel or OGX011, respectively, as part of trial protocols.

Pathological parameters combined with preoperative clinical stage are shown in Table 2. One-hundred fourteen $(39.6 \%)$ patients had organ-confined disease (pT2N0, margin negative), 75 (26\%) had specimen-confined disease (pT3N0, margin negative), $76(26.4 \%)$ had non specimen-confined disease (pT2N0 or pT3N0, margin positive) and $23(8 \%)$ had positive lymph nodes. Of the 156 patients with pT3N0 disease, 68 (43.6\%) had seminal vesicle involvement and $73(46.8 \%)$ had a positive margin.

Of the clinical T2 and T3 patients who did not receive neoadjuvant ADT, 105 had available data for both biopsy and specimen pathology. When Gleason scores were grouped as $<7$, 7 and $>7$, biopsy and specimen Gleason scores were the same in $52.3 \%$, upgraded in the specimen in $15 \%$ and downgraded in the specimen in $32.7 \%$ of patients. Interestingly, $16.5 \%$ of those with Gleason scores 8 or higher were downgraded to Gleason score 7 and no longer classifier as high risk. Similarly, $39.7 \%$ of the cT3 patients were organ confined, illustrating that a significant minority of high-risk patients are downgraded with surgery to intermediate-risk disease.

\begin{tabular}{lcccc}
\hline \multicolumn{5}{l}{ Table 1. Baseline characteristics } \\
\hline \multicolumn{7}{c}{ All } & 1 Risk factor & $\begin{array}{c}\text { 2 Risk } \\
\text { factors }\end{array}$ & $\begin{array}{c}\text { 3 Risk } \\
\text { factors }\end{array}$ \\
\hline No. & 299 & 244 & 47 & 8 \\
Age at & 63.3 (range & 63.4 (range & 64.2 (range & 60.2 (range \\
surgery & $46.2-75.9)$ & $46.1-75.9)$ & $49.9-73.4)$ & $46.1-67.1$ ) \\
Diagnostic & 10 (range & 9.05 (range & 22.8 (range & 25 (range \\
PSA & $0.6-310)$ & $0.6-310)$ & $3.17-47.4)$ & $25-42.9$ ) \\
cT1c & 80 & 132 & 6 & $/$ \\
cT2 & 145 & 74 & 13 & $/$ \\
cT3 & 73 & 37 & 28 & 8 \\
cT4 & 1 & 1 & $/$ & $/$ \\
Gleason $<7$ & 31 & 29 & 2 & $/$ \\
Gleason 7 & 68 & 61 & 7 & $/$ \\
Gleason $>$ 7 & 200 & 154 & 38 & 8 \\
\hline PSA: prostate-specific antigen. & & &
\end{tabular}


Koupparis et al.

\begin{tabular}{lcccc}
\hline \multicolumn{5}{l}{$\begin{array}{l}\text { Table 2. Postoperative pathology and margin status for } \\
\text { all clinical stages }\end{array}$} \\
\hline Clinical stage & cT1c & cT2 & cT3 & cT4 \\
\hline All & 80 & 145 & 73 & 1 \\
Margin positive & $24(30 \%)$ & $49(33.8 \%)$ & $20(27.4 \%)$ & 1 \\
Margin negative & $56(70 \%)$ & $88(60.7 \%)$ & $53(72.6 \%)$ & 0 \\
Lymph node positive & $5(6.25 \%)$ & $13(9 \%)$ & $4(5.5 \%)$ & 0 \\
Lymph node negative & $71(88.75 \%)$ & $125(86.2 \%)$ & $69(94.5 \%)$ & 1 \\
$\begin{array}{l}\text { Seminal vesicle } \\
\text { positive }\end{array}$ & $12(15 \%)$ & $33(22.8 \%)$ & $23(31.5 \%)$ & 1 \\
$\begin{array}{l}\text { Seminal vesicle } \\
\text { negative }\end{array}$ & $64(85 \%)$ & $102(70.3 \%)$ & $50(68.5 \%)$ & 0 \\
\hline Data incomplete for 14 patients. & & & & \\
\hline
\end{tabular}

The recurrence rates according to baseline PSA, Gleason score, clinical stage and number of risk factors are shown in Table 3. The OS, CSS and BPFS rates were 99\%, 99.67\% and $70.23 \%$, respectively, with a median follow-up of 4.7 years (range 0.5-17.3 years). Table 4 shows these values when stratified according to the number of risk factors.

Of the 87 patients with a PSA recurrence, $64(73.65 \%)$ underwent salvage treatments, 31 (35.6\%) ADT alone, 24 (27.6\%) salvage radiation and ADT and 9 (10.3\%) salvage radiation alone. We illustrate the Kaplan Meier curves BPFS for all patients and also curves for the number of risk factors (Fig. 1).

On univariate analysis, PSA at diagnosis, percentage of cores positive and number of risk factors are predictors of PSA recurrence $(p<0.05)$. On multivariate analysis, PSA at diagnosis is the only predictor of PSA recurrence (Table 5).

\section{Discussion}

The optimal management of high-risk prostate cancer remains controversial, with gradual evolution towards mul-

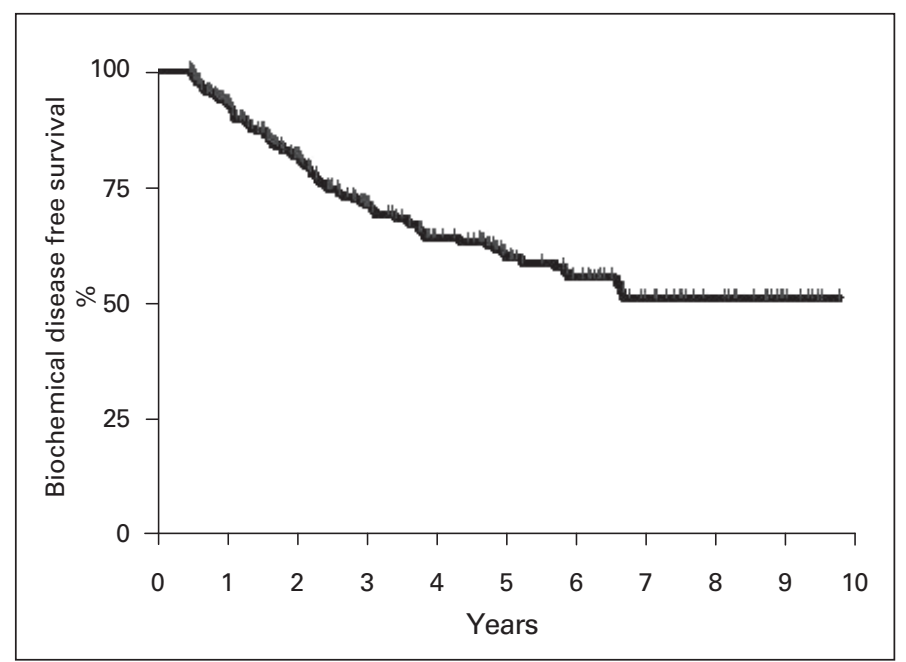

Fig. 1a. Kaplan-Meier curve of prostate-specific antigen recurrence in all patients. timodal therapies. Several studies demonstrate that $\mathrm{RT}$ in combination with ADT is superior to RT alone for locally advanced disease, ${ }^{4}$ and has therefore become a standard of care for these patients. However, there is a lack of data demonstrating that the combination of RT and ADT is superior to RP. Surgery has been shown to

\begin{tabular}{|c|c|}
\hline Variable & Recurrence, no. (\%) \\
\hline PSA $<10$ & $30(20.4 \%)$ \\
\hline PSA $10-20$ & 22 (35.5\%) \\
\hline PSA $>20$ & 35 (38.9\%) \\
\hline Gleason $<7$ & $10(32.3 \%)$ \\
\hline Gleason 7 & $22(32.4 \%)$ \\
\hline Gleason $>7$ & $55(27.5 \%)$ \\
\hline cT1 & $17(21.3 \%)$ \\
\hline cT2 & $41(28.3 \%)$ \\
\hline cT3 & $28(38.4 \%)$ \\
\hline cT4 & $1(100 \%)$ \\
\hline 1 Risk factor & $60(24.6 \%)$ \\
\hline 2 Risk factors & $22(46.8 \%)$ \\
\hline 3 Risk factors & $5(62.5 \%)$ \\
\hline
\end{tabular}
be more beneficial versus watchful waiting in terms of mortality, risk of local progression and risk of metastases; ${ }^{16}$ these benefits became evident after 8 years of follow-up. These observations suggest a positive treatment effect on high-risk prostate cancer patients as low-risk patients have a more protracted natural history and require more than 8 years for risk of death from cancer. In other high-risk localized cancers, notably breast and colorectal cancers, surgery followed by adjuvant or salvage treatments is either recommended or commonly employed (in accordance with the NCCN guidelines).

Some centres have adopted a multimodal approach to these high-risk patients, with primary RP and adjuvant or salvage RT where appropriate. Several published surgical series of high-risk localized prostate cancer patients demonstrate OS and clinical progression free survival (CPFS) rates which surpass $\mathrm{RT}$ alone and similar to RT combined

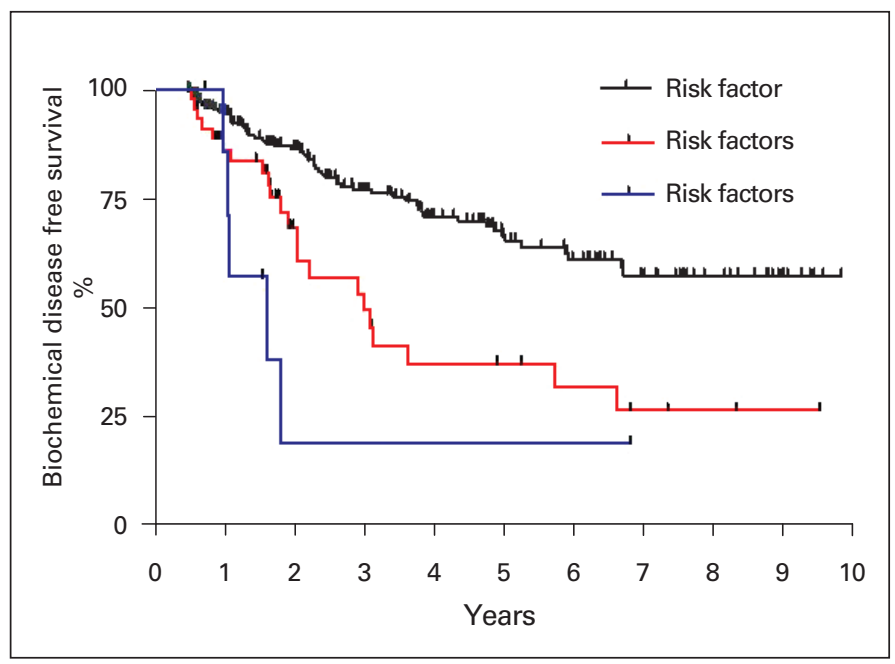

Fig. 1b. Kaplan-Meier curve of prostate-specific antigen stratified according number of risk factors. 


\begin{tabular}{lcccc}
\hline $\begin{array}{l}\text { Table 4. Overall, cancer-specific, and biochemical disease- } \\
\text { free survival }\end{array}$ & $\begin{array}{c}\text { Median time to } \\
\text { recurrence (mos) }\end{array}$ & OS (\%) & CSS (\%) & BPFS (\%) \\
\hline All & $25.1(2.4-144.5)$ & 99 & 99.67 & 70.23 \\
1 Risk factor & $27.5(2.4-144.5)$ & 98.77 & 99.59 & 75.41 \\
2 Risk factors & $22.8(4.3-80.5)$ & 100 & 100 & 53.19 \\
3 Risk factors & $13.2(12.3-22.2)$ & 100 & 100 & 37.5 \\
\hline
\end{tabular}

OS: overall survival, CSS: cancer-specific survival; BPFS: biochemical progression-free survival.

with ADT.7,9,17 Moreover, several randomized trials have demonstrated that immediate postoperative RT after RP with adverse pathology reduces PSA recurrence rates ${ }^{18-20}$ and improves metastasis-free and OS rates..$^{21}$ Multimodal therapy, with an initial RP, may therefore offer equivalent rates of cancer control compared to RT in these patients, without the need for long-term adjuvant ADT.

The present study demonstrates an OS, CSS and BPFS of $99 \%, 99.67 \%$ and $70.23 \%$, respectively, with a median follow-up of 4.7 years. This finding is consistent with other published series using surgical monotherapy or multimodal therapy. Ten-year outcome data was published by Donohue and colleagues on 238 patients with biopsy Gleason scores 8 to 10 who received RP as their only treatment. ${ }^{22}$ The authors concluded that these patients do not have a uniformly poor outcome and $39 \%$ are recurrence-free at 10 years.

In terms of multimodal therapy, the Mayo clinic published the largest surgical series to date, with 842 patients with clinical T3 disease and a median follow-up of more than 10 years who underwent RP. ${ }^{7}$ The BPFS and CPFS were $58 \%$ and $85 \%$ at 5 years and $43 \%$ and $73 \%$ at 10 years, respectively. Fifty-one percent of patients received adjuvant ADT and $16 \%$ underwent adjuvant RT. Hsu and colleagues analyzed 235 clinical T3 prostate cancer patients and found a similar BPFS of $59.5 \%$ and $51.5 \%$ at 5 and 10 years, respectively, and an even higher CPFS than the Mayo series of $96 \%$ and $85 \%$ at 5 and 10 years, respectively. ${ }^{9}$ Of these patients, $56 \%$ had either adjuvant or salvage RT and/or ADT. More recently, Loeb and colleagues examined 175 high-risk patients who underwent RP by a single surgeon. At 10 years, the BPFS was 68\%, metastasis-free survival was $84 \%$ and CSS was $92 \% .{ }^{17}$ The 10 -year rate of freedom from any hormonal therapy was $71 \%$. Of the high-risk criteria, a biopsy Gleason score of 8 to 10 (vs. s7) was the strongest independent predictor of biochemical recurrence, metastases and prostate cancer death.

These figures appear to afford similar or superior OS and CPFS when compared to RT plus ADT-based regimens commonly employed for these patients. The BPFS is however lower in the surgical series because of definitions employed for PSA recurrence, as well as the uniform use of longterm ADT, which can suppress detection of this androgenregulated biomarker. In the combination therapy arm of the European Organisation for Research and Treatment of Cancer (EORTC) 22863 trial, the 203 patients receiving RT plus 3 years of ADT (91\% of whom had clinical T3 disease) had a clinical BPFS of $76 \%$ at 5 years. ${ }^{4}$ However, the definition of PSA recurrence was much less stringent, at $1.5 \mathrm{ng} / \mathrm{mL}$ and increasing on 2 consecutive measurements.

In addition to equivalent or better PFS outcomes, RP as the primary treatment may offer several other advantages over primary RT. As many as $27 \%$ of patients identified as having cT3 disease are downstaged to pT2 disease. ${ }^{7}$ In the present study, $30 \%$ of all patients were downgraded, and of those cT3 patients 29 (39.7\%) were subsequently downstaged to organ-confined disease. Only RP can provide pathological staging to discern these patients, in whom monotherapy may be the only treatment required, thereby avoiding overtreatment with RT plus 3 years of ADT which is associated with worse outcomes across multiple quality of life domains in patients. ${ }^{23}$

A pelvic lymph node dissection should be performed during RP for high-risk prostate cancer, as $15 \%$ to $40 \%$ will be positive. ${ }^{24}$ If positive lymph nodes are found, ADT can be commenced, which has been shown, albeit in the pre-PSA era, to improve survival. ${ }^{25}$

High-risk patients may subsequently experience local treatment failure and require adjuvant or salvage therapies. ${ }^{26}$ The rate of long-term complications can be affected by the order in which treatments are given. Surgery, as the primary local treatment, is associated with a lower complication rate and smaller impact on quality of life when compared

Table 5. Predictors of PSA recurrence using univariate and multivariate analysis

\begin{tabular}{lcccc}
\hline Parameters & $\begin{array}{c}\text { Univariate analysis } \\
\boldsymbol{p} \text { value (Welch } \\
\text { 2-sample T-test) }\end{array}$ & $\begin{array}{c}\text { Univariate analysis } \\
\boldsymbol{p} \text { value (Wilcoxon } \\
\text { rank sum test with } \\
\text { continuity correction) }\end{array}$ & $\begin{array}{c}\text { Multivariate analysis } \\
\boldsymbol{p} \text { value (excluding } \\
\text { pathological stage and } \\
\text { risk number) }\end{array}$ & $\begin{array}{c}\text { Multivariate analysis } \\
\boldsymbol{p} \text { value (excluding } \\
\text { pathological stage but } \\
\text { not risk number) }\end{array}$ \\
\hline PSA at diagnosis & 0.03662 & 0.01910 & 0.002403 & 0.011257 \\
Biopsy Gleason score & 0.06489 & 0.06601 & 0.014019 & 0.083551 \\
Percentage of cores positive & 0.03315 & 0.04027 & 0.175520 & 0.404776 \\
Clinical T stage & 0.3895 & 0.3810 & 0.650240 & 0.782646 \\
No. risk factors & 0.01238 & 0.002627 & & \\
\hline PSA: prostate-specific antigen. & & & & \\
\hline
\end{tabular}


Koupparis et al.

to salvage RP after RT. ${ }^{27}$ Rates of urinary incontinence and stricture after salvage RP have been reported as $30 \%$ to $66 \%$ and $28 \%$, respectively. ${ }^{28}$ These findings compare to data from patients receiving adjuvant RT following RP, which demonstrate rates of total urinary incontinence and urethral stricture of $6.5 \%$ (vs. $2.8 \%$ ) and $18 \%$ (vs. $10 \%$ ), respectively. ${ }^{21}$

The morbidity of RP in patients with high-risk disease appears no greater than in those patients with localized disease, ${ }^{7}$ and the 30 -day mortality in the current series was 0 . However, the maintenance of erectile function in these patients is low; $;, 9$ in the current study, $75.2 \%$ patients reported erectile dysfunction as a result of the wide resection required in high-risk disease. The rates of erectile dysfunction do, however, compare favourably to those after RT for clinical T3 disease. ${ }^{29}$

A further benefit of primary RP in patients with high-risk clinically localized prostate cancer may be in the subsequent development of metastatic disease. The Southwest Oncology Group (SWOG) 8894 compared orchidectomy to orchidectomy plus flutamide in patients with metastatic disease. Based on the improved survival seen in patients with metastatic renal and ovarian cancer whose primary tumour has been removed, Thompson and colleagues re-analyzed the data from this trial. ${ }^{30}$ Although only hypothesis-generating, they found a reduced risk of death in patients with metastatic disease if they had undergone prior RP compared to patients who had no previous definitive therapy (hazard ratio [HR] 0.77). Conversely, patients who had undergone prior RT had an increased risk of death (HR 1.22).

Our study has several limitations. Firstly, as a single institution series of a tertiary referral centre, it may not reflect outcomes achieved in the broader clinical community. Secondly, there was no central pathology review of biopsies within our institution. This may account for the $30 \%$ downgrading seen from biopsies to specimen pathology. Whereas $62.7 \%$ of all biopsies were reported as Gleason score 8 to 10 , only $31.8 \%$ of evaluable surgical specimens were reported as such, suggesting that biopsies may have been overgraded. However, previous series have shown up to $45 \%$ of biopsy-Gleason scores 8 to 10 were downgraded in the surgical specimen, despite central review of the biopsies by dedicated uropathologists. ${ }^{22}$ Thirdly, a significant proportion of patients received neoadjuvant therapies as part of trial protocols which may affect the discussed outcomes. However, a Cochrane review published in 2006 concluded that neoadjuvant ADT improves organ-confined rates, pathological downstaging, positive surgical margins and rate of lymph node invasion, ${ }^{31}$ but neither neoadjuvant nor adjuvant ADT before RP provides a significant OS or disease-free survival advantage over RP alone. However, in a randomized study of 3 months versus 8 months of neoadjuvant ADT prior to RP biochemical, no evidence of disease rates were significantly lower in the 267 men enrolled at the 3 top-recruiting "high volume" sites (24\%) compared to 238 men enrolled at the remaining 8 "low volume" sites $(40 \%)(p<0.001) .{ }^{12}$ Furthermore, the margin positive rate and lymph node invasion rate for patients who received neoadjuvant therapies in the present study are $25.2 \%$ and $6 \%$, respectively (vs. $26.4 \%$ and $8 \%$ overall).

\section{Conclusions}

The present study demonstrates OS, CPFS and BPFS similar to other surgical series and comparable to RT combined with ADT. These data support recommendation of RP as treatment option for patients with high-risk prostate cancer. Radical prostatectomy followed, where appropriate, by adjuvant or salvage RT has several benefits. It limits overtreatment of those patients who are initially overstaged, avoids the long-term side-effects of ADT, allows selective adjuvant and salvage treatments to lower risk of complications and identifies patients with lymph node positive disease who may benefit from early treatment.

The Vancouver Prostate Centre, Department of Urological Sciences, University of British Columbia, Vancouver, $\mathrm{BC}$

Competing interests: None declared.

This paper has been peer-reviewed.

\section{References}

1. Makarov DV, Trock BJ, Humphreys EB, et al. Updated nomogram to predict pathologic stage of prostate cancer given prostate-specific antigen level, clinical stage, and biopsy Gleason score (Partin tables) based on cases from 2000 to 2005. Urology 2007;69:1095-101.

2. Cooperberg MR, Lubeck DP, Mehta SS, et al. Time trends in clinical risk stratification for prostate cancer: implications for outcomes (data from CaPSURE). J Urol 2003;170(6 Pt 2):S21-5; discussion S26-7.

3. Fallon B, Williams RD. Current options in the management of clinical stage C prostatic carcinoma. Urol Clin North Am 1990;17:853-66.

4. Bolla M, Collette L, Blank L, et al. Long-term results with immediate androgen suppression and external irradiation in patients with locally advanced prostate cancer (an EORTC study): a phase III randomised trial. Lancet 2002;360:103-6.

5. Laverdiere J, Gomez JL, Cusan L, et al. Beneficial effect of combination hormonal therapy administered prior and following external beam radiation therapy in localized prostate cancer. Int I Radiat Oncol Biol Phys 1997;37:247-52.

6. Denberg TD, Glode LM, Steiner JF, et al. Trends and predictors of aggressive therapy for clinical locally advanced prostate carcinoma. BJU Int 2006;98:335-40.

7. Ward JF, Slezak JM, Blute ML, et al. Radical prostatectomy for clinically advanced (CT3) prostate cancer since the advent of prostate-specific antigen testing: 15-year outcome. BJU Int 2005;95:751-6.

8. Berglund RK, Jones IS, Ulchaker JC, et al. Radical prostatectomy as primary treatment modality for locally advanced prostate cancer: a prospective analysis. Urology 2006;67:1253-6.

9. Hsu CY, Joniau $S$, Oyen R, et al. Outcome of surgery for clinical unilateral T3a prostate cancer: a singleinstitution experience. Eur Urol 2007;51:121-8; discussion 8-9.

10. Scardino P. Update: NCCN prostate cancer Clinical Practice Guidelines. J Natt Compr Canc Netw 2005;3 (Suppl 1):S29-33. 
$\mathrm{RP}$ for high-risk localized prostate cancer

11. Yossepowitch 0, Eggener SE, Bianco FJ Jr, et al. Radical prostatectomy for clinically localized, high risk prostate cancer: critical analysis of risk assessment methods. J Urol 2007;178:493-9; discussion 499.

12. Gleave ME, Goldenberg SL, Chin JL, et al. Randomized comparative study of 3 versus 8-month neoadjuvant hormonal therapy before radical prostatectomy: biochemical and pathological effects. J Urol 2001;166:500-6; discussion 506-7.

13. Chi KN, Chin JL, Winquist E, et al. Multicenter phase II study of combined neoadjuvant docetaxel and hormone therapy before radical prostatectomy for patients with high risk localized prostate cancer. I Urol 2008: 180:565-70; discussion 570

14. Chi KN, Eisenhaver E, Fazli L, et al. A phase I pharmacokinetic and pharmacodynamic study of OGX-01 I, a 2'-methoxyethyl antisense oligonucleotide to clusterin, in patients with localized prostate cancer. J Natl Cancer Inst 2005;97:1287-96.

15. Amling $\mathrm{CL}$, Bergstralh EJ, Blute ML, et al. Defining prostate specific antigen progression after radical prostatectomy: what is the most appropriate cut point? J Urol 2001;165:1146-51.

16. Bill-Axelson A, Holmberg L, Ruutu M, et al. Radical prostatectomy versus watchful waiting in early prostate cancer. N Engl J Med 2005;352:1977-84.

17. Loeb S, Schaeffer EM, Trock BJ, et al. What are the outcomes of radical prostatectomy for high-risk prostate cancer? Urology 2010 Sep; 76(3):710-4. Epub 2009 Nov 22.

18. Thompson IM Jr, Tangen CM, Paradelo J, et al. Adjuvant radiotherapy for pathologically advanced prostate cancer: a randomized clinical trial. JAMA 2006;296:2329-35.

19. Bolla $M$, van Poppel $H$, Collette $L$, et al. Postoperative radiotherapy after radical prostatectomy: a randomised controlled trial (EORTC trial 22911). Lancet 2005;366:572-8.

20. Wiegel T, Bottke D, Steiner U, et al. Phase III postoperative adjuvant radiotherapy after radical prostatectomy compared with radical prostatectomy alone in $\mathrm{p} T 3$ prostate cancer with postoperative undetectable prostate-specific antigen: ARO 96-02/AUO AP 09/95. J Clin Oncol 2009;27:2924-30.

21. Thompson IM, Tangen CM, Paradelo J, et al. Adjuvant radiotherapy for pathological T3NOMO prostate cancer significantly reduces risk of metastases and improves survival: long-term followup of a randomized clinical trial. J Urol 2009;181:956-62.
22. Donohue JF, Bianco FJ Jr, Kuroiwa K, et al. Poorly differentiated prostate cancer treated with radical prostatectomy: long-term outcome and incidence of pathological downgrading. J Urol 2006;176:991-5.

23. Sanda MG, Dunn RL, Michalski J, et al. Quality of life and satisfaction with outcome among prostate-cancer survivors. N Engl J Med 2008;358:1250-61.

24. Briganti A, Chun FK, Salonia A, et al. Validation of a nomogram predicting the probability of lymph node invasion based on the extent of pelvic lymphadenectomy in patients with clinically localized prostate cancer. BJU Int 2006:98:788-93.

25. Messing EM, Manola J, Sarosdy M, et al. Immediate hormonal therapy compared with observation after radical prostatectomy and pelvic lymphadenectomy in men with node-positive prostate cancer. N Engl J Med 1999;341:1781-8.

26. Swanson GP, Hussey MA, Tangen $C M$, et al. Predominant treatment failure in postprostatectomy patients is local: analysis of patterns of treatment failure in SWOG 8794. I Clin Oncol 2007;25:2225-9.

27. Tefilli MV, Gheiler EL, Tiguert R, et al. Quality of life in patients undergoing salvage procedures for locally recurrent prostate cancer. J Surg Oncol 1998;69:156-61.

28. Chen BT, Wood DP Jr. Salvage prostatectomy in patients who have failed radiation therapy or cryotherapy as primary treatment for prostate cancer. Urology 2003;62(Suppl 1):69-78.

29. Fransson P, Lund JA, Damber JE, et al. Quality of life in patients with locally advanced prostate cancer given endocrine treatment with or without radiotherapy: 4-year follow-up of SPCG-7/SFU0-3, an openlabel, randomised, phase III trial. Lancet Oncol 2009;10:370-80.

30. Thompson IM, Tangen C, Basler J, et al. Impact of previous local treatment for prostate cancer on subsequent metastatic disease. J Urol 2002;168:1008-12.

31. Kumar S, Shelley M, Harrison C, et al. Neo-adjuvant and adjuvant hormone therapy for localized and locally advanced prostate cancer. Cochrane Database Syst Rev 2006:CD006019.

Correspondence: Dr. Anthony J. Koupparis, The Vancouver Prostate Centre, Department of Urological Sciences University Of British Columbia, Gordon \& Leslie Diamond Health Care Centre Level 6, 2775 Laurel St., Vancouver, BC V5Z 1M9; anthonykoupparis@googlemail.com 